\title{
Test system of myoelectric signals to measure voltages to hand prosthesis
}

\begin{abstract}
The electromyographic signals (EMG) also known as myoelectric signals, occur with movements of contraction and distension of muscles. These can be generated on a voluntary way; they are widely used for their functionality to control man - machine interfaces. During recent years it has been sought to figure out of these signals for the development of arm or leg prostheses. In the present research, a system of capturing, through the amplification and filtering of myoelectric signals, it was implemented a test system control in a hand prosthesis. The outcomes of the measurements of the voltages generated, had shown the performing of a grip prehensile, also can be infer the validate signal with computational tools to verify the correct operation of the proposed system and have a more faithful and stable parameterization.
\end{abstract}

Keywords: electromyographic signals, embedded system grip prehensile, bioelectrical signals
Volume 3 Issue 5 - 2019

\author{
Piña Díaz AJ,Torres San Miguel CR, \\ Hernández Rodríguez MA, Romero Ángeles \\ B, Urriolagoitia Sosa G, Urriolagoitia \\ Calderón GM \\ Instituto Politécnico Nacional, México
}

Correspondence: Piña Díaz AJ, Instituto Politécnico Nacional, Postgraduate Studies and Research Section of the Higher School of Mechanical and Electrical Engineering, México, Email armpi07@hotmail.com

Received: October 15, 2019 | Published: October 22, 2019

\section{Introduction}

In the human body different bioelectrical signals are generated, which are presents in tissues, cells, nerves, glands, among others. These types of signals allow the functioning of organs such as; the brain, the heart, the eyes, the muscles, etc. The bioelectrical signals are given characteristic names depending on the organ in which they originate. ${ }^{1}$ The main limbs for the physical manipulation of the medium are the hands, where the fingertips contain some of the areas with the most nerve endings of the human body. This upper extremity has been a key partner of the brain to convert thought into action, in it, ideas are translated mechanically into actions, creating hierarchical representations to configure the processes necessary in the control of movements and manipulation of objects. ${ }^{2}$ The clinical use of EMG signals for the treatment of diseases began in the 1960s. Hardyck and his colleagues were the first practitioners to apply the EMG. ${ }^{3}$ In the early 80 's Cram and Steger introduced a clinical method for scanning a variety of muscles using sensitive EMG device. ${ }^{4}$

It was not until the middle of the 80 's, when electrode techniques were integrated, which had a technological level sufficient for the production of small and low weight instrumentation. Also, amplifiers that allowed advances in fields such as Medicine or Biomechanics. ${ }^{5}$ Electromyography has been widely used for the recording of superficial muscles in clinical protocols or kinesiologists. Where intramuscular electrodes are used to investigate deep muscles or locate muscle activity. ${ }^{6}$ There are currently a number of applications for the use of EMG signals. For example, it is used clinically for the diagnosis of neural or neuromuscular problems, for the analysis of motor control, neuromuscular physiology, movement disorders, postural control, and physical therapy. ${ }^{7}$ Not all studies related to myoelectric signals are focused on their use in prostheses; some of them are dedicated to taking samples through the devices of acquisition of these signals, monitoring and parameterization to detect certain types of diseases. ${ }^{8}$ Some studies on the detection of biological signals also focus on the detection of textures as proposed in ${ }^{9}$ and it could help in the development of bionic prostheses, adapting these techniques to improve the functionality of these prostheses.

As a result of the increase in studies on EMG signals, in Mexico research has been carried out, such as that carried out in 2013 by ÁvilaMorales et al., ${ }^{10}$ Which designed and constructed an electromechanical prosthesis of a human hand controlled by EMG signals. ${ }^{10}$ This leaves an open panorama on the possibilities of development in the implementation of the electromyographic signals.

Given the need for a correct analysis of myoelectric signals, the present work developed the algorithm necessary for the acquisition and processing of the signals produced by the muscles of the forearms involved in the process of hand movement when performing the prehensile grip.

Taking this study as a preamble for control systems capable of giving the highest possible precision, these signals can be used in the generation of movements in previously designed active hand prosthesis, Figure 1.

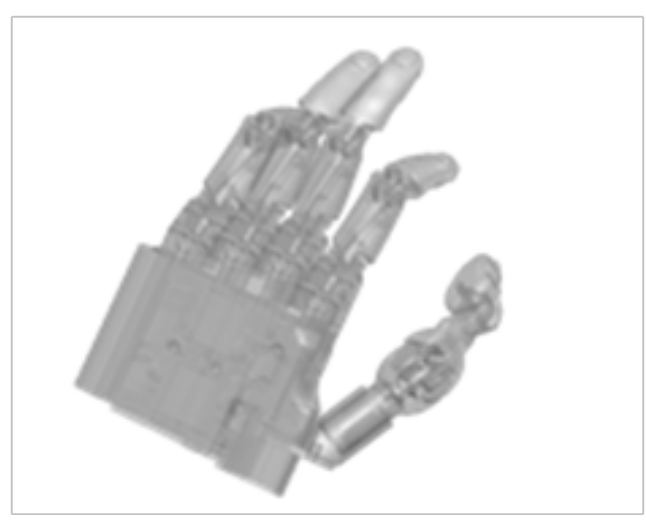

Figure I Pre-developed robotic prosthesis design. 


\section{Material and methods}

The myoelectric signals are signals, produced by contraction and relaxation of any muscle, according to this characteristic was developed an analysis of the myoelectric signals of a 25 -year-old middle-aged patient with a weight of $80 \mathrm{~kg}$ and a $\mathrm{pH} 6.5$. The classification phase, within the process of analysis of these signals or pattern recognition, is a very important component and can be considered as the final task of the process, since once the experiment has been defined, the signals obtained and preprocessed, the last step is the implementation of a classifier that can decide which class corresponds to each of them. And with these signals can simulate the movements of the fingers of the prosthesis of the hand, and thereby supplement the lack of the limb with a useful mechanism.

In the development of this research for the acquisition of the signals were used surface electrodes RedDot ${ }^{\mathrm{TM}} 2239$ model of the $3 \mathrm{M}^{\mathrm{TM}}$ brand, connected to a Tektronix ${ }^{\circledR}$ TDS 1002 digital oscilloscope. In signal processing, a circuit was designed to amplify and clean the EMG signals, using an instrumentation amplifier AD620; and an integrated circuit (IC) TL074. The complete EMG signal conditioning circuit; as well as all other circuits of the individual stages, were simulated in the NI Multisim ${ }^{\mathrm{TM}} 13.0$ program of National Instruments ${ }^{\mathrm{TM}}$, to verify the correct operation of the electronic device designed, prior to its manufacture. There are different ways of achieve the acquisition of the EMG signal in the various types of muscles, either invasively using needle electrodes directly grafted onto the muscle to be monitored, or noninvasively using surface electrodes, ${ }^{11}$ taking into consideration that the number of electrodes corresponds directly to the number of channels to be processed and consequently to the number of movements to be modeled..$^{12}$

It is important to consider the different stages of filtering and amplification in order to obtain the most accurate and undisturbed EMG signal and to have an easy-to-read signal for the control devices. For this investigation the EMG signals are collected by surface bipolar electrodes, which are located on the skin, where a gel was applied that improved the conductivity and adhesion with the electrodes. ${ }^{13}$ Despite these arrangements, the collected signals will be too weak, so a preprocessing of filtering and amplification is necessary before analysis. These aspects are detailed in Figure 2. In accordance with Sarmiento in ${ }^{14}$ the activator muscle must be identified with the artificial electrodes in such a way that a reference threshold is reached in conjunction with the reference. In addition, Dorador $\mathrm{in}^{15}$ mentions that the location of the surface electrodes for the capture of myoelectric signals should be in the area of the biceps.

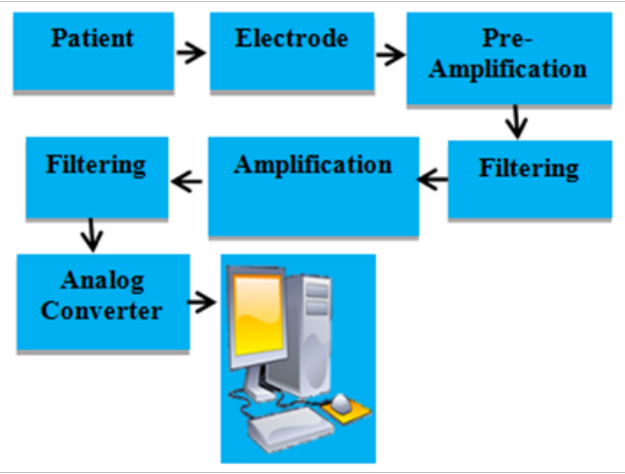

Figure 2 Proposed system for the acquisition of EMG signals.
During the amplification stage of the captured myoelectric signals, according to previous studies an amplification range of at least 500 to 1000 times should be considered. ${ }^{16}$ According to SENIAM regulations ${ }^{17}$ it is recommended that surface bipolar electrodes have an average separation distance between centers of between $20 \mathrm{~mm}$ and $30 \mathrm{~mm}$ and that if placed on relatively small muscles, the distance between electrodes should not exceed 1/4 of the length of the muscle fiber. It is also important to consider the type of movement performed, because isometric movements and exercises that require static positions obtained signal should be considered under large time conditions (usually $>150 \mathrm{~ms}$ ), while dynamic movements the time constants analyzed should Be chosen with an average of $60 \mathrm{~ms}$ or less. ${ }^{18}$

Considering the locations proposed in the literature, 3 surface electrodes were placed on the forearm as shown in Figure 3. To decrease possible factors affecting the capture of EMG signals, such as sweat, beauty and variation of the impedance of the patient's skin. Readings of the EMG signals were collected for flexing all the fingers together, spherical prehensile grips, motivating the patient to close the fist to the maximum degree and obtaining EMG pulse measurement generated in the following ways: during relaxation of the muscle, with a pulse of the muscle and the gradual increase of the force in the closure of the hand.

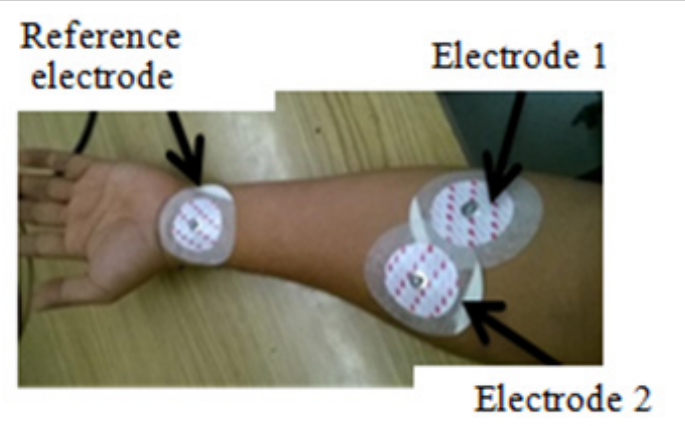

Figure 3 Electrode positioning.

The signal acquisition block consists of transducers and, in particular, electrodes that convert the myoelectric signal generated in the arm muscle into an electrical signal to be processed. Figure 4 shows the procedure for obtaining myoelectric signals using the oscilloscope. The block that serves to adapt the signals, is composed of three different stages, in the first stage of pre-amplification, where an instrumentation amplifier AD620 was used, which has the task of acquiring the differential signal of the electrodes connected to the patient's body. Subsequently, this signal was filtered through a oneorder Butterworth band pass filter, as well as a high pass filter and a low pass filter. Finally, amplify the signal by the relation that exists through the gain resistance. By means of the instrumentation amplifier AD620 (Figure 5), the signals were obtained with a high impedance in differential form.

For the design of the filtering stage it was necessary to use a preamplification stage which is achieved with a TL074 CI. Figure 6 shows the electronic circuit of the pre-amplification stage. And the simulation of its function with a generated signal of $200 \mathrm{mV}$, by reason of a frequency of $5 \mathrm{~Hz}$, by means of a generator of functions with a sine wave is shown Figure 7, which simulated the muscle to the maximum effort, according to the measurements taken In the exercise of capture of the electromyographic signals, for demonstrative purposes of the operation of the pre-amplification stage. 


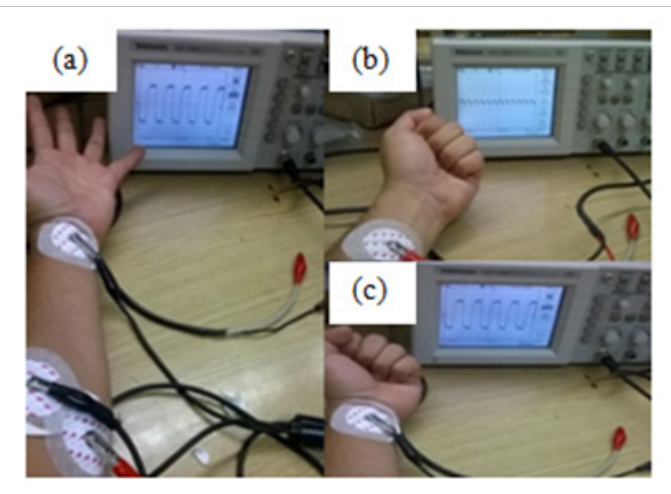

Figure 4 Reading of EMG signals, with muscle at rest (a), gradual exertion (b) and maximal muscle effort (c).

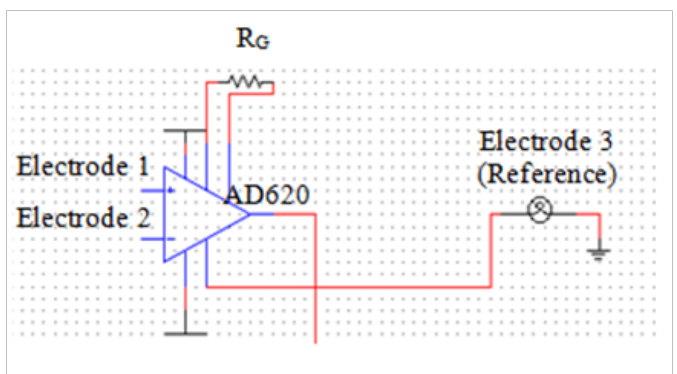

Figure 5 Instrumentation amplifier diagram.

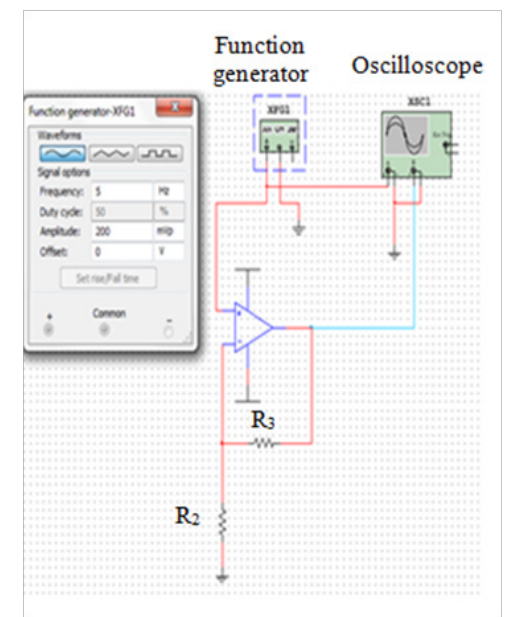

Figure 6 Diagram of the pre-amplification stage.

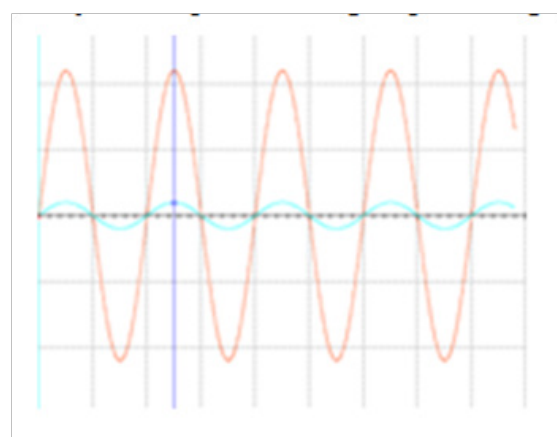

Figure 7 Signal obtained at the output of the instrumentation pre-amplifier stage (red), compared to the input signal (blue).
In Figure 7 , the input signal with a peak of $200 \mathrm{mV}$, reaches a peak of $2.2 \mathrm{~V}$, after the amplification process, which makes it more recognizable to the filtering process and to the control devices that are will eventually use the process of signal processing. Due to the possible interference produced in the environment, the optimum frequency range will be from 20 to $500 \mathrm{~Hz}$. In order to limit the frequency, range between 0 and $500 \mathrm{~Hz}$, a Butterworth bandpass filter of order one was used. It is known that the useful range of measurement is between 10 and $20 \mathrm{~Hz}$, so a high pass filter was used, and up to $500 \mathrm{~Hz}$ and for less than $1500 \mathrm{~Hz}$, a low pass filter. ${ }^{19,20}$ The high pass filter is necessary to eliminate noise from nearby muscles, and other muscle movements (artifacts) as well as repolarization potentials of muscles having low frequency components, typically $<10 \mathrm{~Hz}$. The low pass filter was necessary for eliminate high frequency components and avoid the generation of false frequencies (aliasing). Figure 8 shows the diagram of the filtering stage of the system.

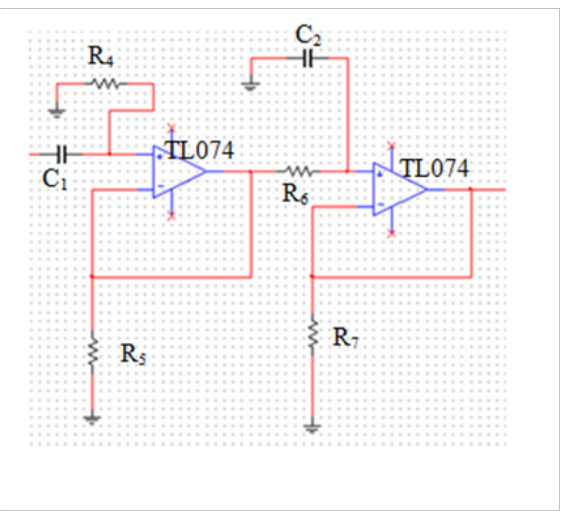

Figure 8 Diagram of the filtering stage of the electromyographic signals.

Once the filtered signal was obtained, Figure 9, a new amplification stage was performed by means of a TL074 circuit, which was simulated with the National Instruments ${ }^{\mathrm{TM}}$ NI Multisim ${ }^{\mathrm{TM}} 13.0$ computational tool to verify its operation. This was the final stage of the EMG signal matching process, so the simulation was performed with the complete circuit design of signal acquisition and processing, obtaining the graph for the maximum effort muscle shown in Figure 10.

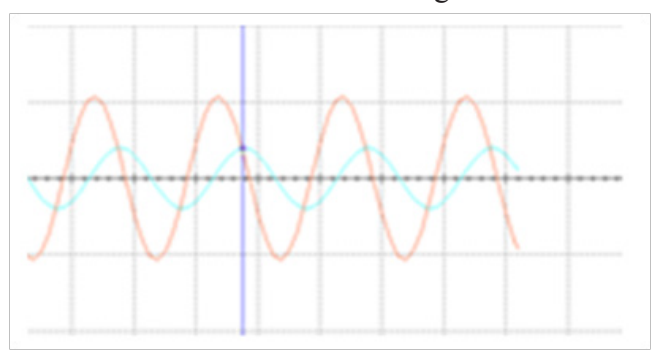

Figure 9 Signal obtained at the output of the filtering step (red), compared to the input signal (blue).

For the test phase of the prototype (Figure 11), the control logic proposed in this research was by means of pulse trains, with which parameters were obtained that establish a logic, to the information that was programmed in the control system to be implemented. AMegaADK Arduino $^{\mathrm{TM}}$ card was used, specifically designed to control servomotors by means of a programming script, looking for the possibility of being modified through of programming of medium level, and with that it would modify parameters such as; Speed, acceleration, freedom of movement of the servos, and even the logic of the electromyographic pulses. 


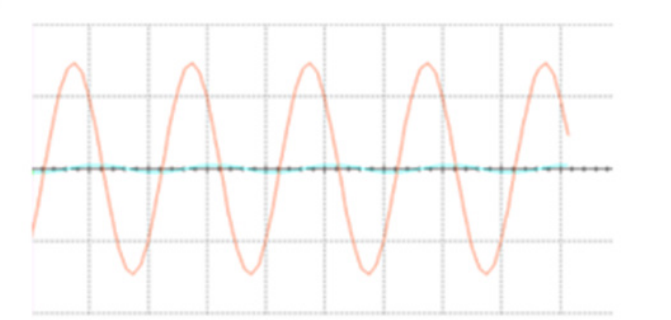

Figure 10 Signal obtained at the output of the final signal processing circuit (red), compared to the input signal (blue).

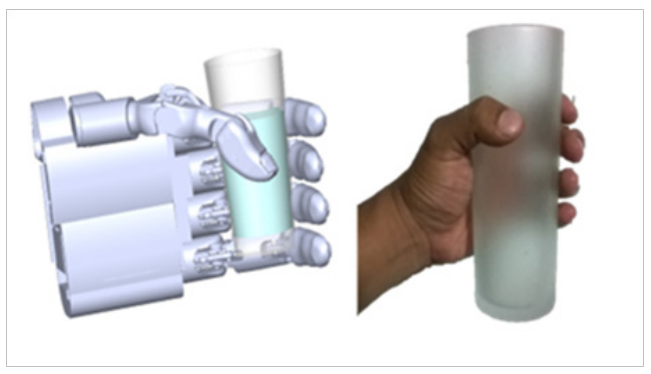

Figure I I Partial prehensile grip emulation, used to hold a glass.

\section{Results}

Following the data obtained from the muscle, the circuit simulation was performed to determine the range of values required for the activation of the servomotors. For the case of the muscle subjected to an average effort has a voltage measured in the muscle of $1.04 \mathrm{~V}$ in the positive zone so that a range of $10 \mathrm{mV} \pm 2 \mathrm{mV}$ in the simulated signal is needed, to obtain that final gain, necessary for the activation of the servomotors. For the case of a pulse in the muscle, it has a voltage measured in the muscle of $3.6 \mathrm{~V}$ in the positive zone with a range of 30 $\mathrm{mV} \pm 2 \mathrm{mV}$ in the simulated signal, to obtain that necessary final gain for the activation of the servomotors.

Finally, for the muscle undergoing a gradual effort there is an average voltage measured of $3 \mathrm{~V}$ in the positive zone so that a range of less than $26 \mathrm{mV}$ in the simulated signal is needed, to obtain that necessary final gain for the activation of the servomotors. The results obtained for the measurement of the electromyographic signal of the muscles of the forearm involved during a flexion movement when performing a complete closure of the hand are shown in Table 1. With the simulations for the muscle in a medium and maximum effort pressure grip, as well as at rest, the following values, were obtained and shown in Table 2.

Table I Data obtained from the reading of EMG signals from the patient's forearm

\begin{tabular}{llll}
\hline Measurement & Resting muscle & Muscle at maximum effort & $\begin{array}{l}\text { Muscle to medium effort } \\
\text { (gradual) }\end{array}$ \\
\hline $\mathrm{VPP}$ & $2.16 \mathrm{~V}$ & $8.40 \mathrm{~V}$ & $6.00 \mathrm{~V}$ \\
$\mathrm{Vmax}$ & $1.04 \mathrm{~V}$ & $3.60 \mathrm{~V}$ & $3.00 \mathrm{~V}$ \\
$\mathrm{Vmin}$ & $-1.12 \mathrm{~V}$ & $-4.80 \mathrm{~V}$ & $-3.00 \mathrm{~V}$ \\
Period & $15.00 \mathrm{~ms}$ & $26.00 \mathrm{~ms}$ & $24.00 \mathrm{~ms}$ \\
Frequency & $66.66 \mathrm{~Hz}$ & $38.46 \mathrm{~Hz}$ & $41.66 \mathrm{~Hz}$ \\
Positive width & $1.67 \mathrm{~s}$ & $17.00 \mathrm{~ms}$ & $628.00 \mathrm{~ms}$ \\
Negative width & $560.00 \mathrm{~ms}$ & $8.00 \mathrm{~ms}$ & $80.00 \mathrm{~ms}$ \\
Upload time & $6.00 \mathrm{~ms}$ & $17.00 \mathrm{~ms}$ & $80.00 \mathrm{~ms}$ \\
Down time & $268.00 \mathrm{~ms}$ & $5.00 \mathrm{~ms}$ & $54.00 \mathrm{~ms}$ \\
Width & $3.77 \mathrm{~s}$ & $42.00 \mathrm{~ms}$ & $815.00 \mathrm{~ms}$ \\
\hline
\end{tabular}

Table 2 Data for simulation of patient EMG signals

\begin{tabular}{llll}
\hline Simulation & Resting muscle & Muscle at maximum effort & $\begin{array}{l}\text { Muscle to medium effort } \\
\text { (gradual) }\end{array}$ \\
\hline $\mathrm{VPP}$ & $2.48 \mathrm{~V}$ & $6.86 \mathrm{~V}$ & $5.858 \mathrm{~V}$ \\
$\mathrm{Vmax}$ & $1.24 \mathrm{~V}$ & $3.43 \mathrm{~V}$ & $2.929 \mathrm{~V}$ \\
$\mathrm{Vmin}$ & $-1.24 \mathrm{~V}$ & $-3.43 \mathrm{~V}$ & $-2.929 \mathrm{~V}$ \\
Simulation frequency & $5 \mathrm{~Hz}$ & $5 \mathrm{~Hz}$ & $5 \mathrm{~Hz}$ \\
\hline
\end{tabular}

\section{Conclusions}

Because of the activation protocol was carried out by pulses, a uniform pulse was made to identify this signal as an activation button for the proposed control system, and it was designed as protection against voltage surges which are generated in the condition of maximum effort in the muscle. For this a circuit known as Schmitt Trigger was implemented, thus this circuit sends an analog signal when it receives an excitation higher than $1.38 \mathrm{~V}$ and maintains it in
$5 \mathrm{~V}$ until that excitation is below $1.38 \mathrm{~V}$. In this way, it was manipulated the signal for activation of the card and at the same time protected from high pulses coming from the effort in the muscle. The signals of the simulation are presented as a complete synodal wave, this since they do not present an external interference, as in the reading of the signals directly in the muscle. In consideration, these values of electrical potentials are observed as a small variation either in the position of the electrode as well as external noise agents that can have a significant influence on the signal obtained. 


\section{Discussions}

Through the presented research, it is shown a methodology that allows to study the myoelectric signals generated in the forearm of a person, resorting for this to the description of the processes that comprise the stages of processing of the signals EMG, from its reading in the muscle, until the final stage of the process of adaptation of the signal for its later use. This prototype of prosthesis (Figure 1) is also developed in this investigation, starting from the simplification of mechanisms and analysis for the direct and inverse kinematics of each of the fingers involved in the grips generated by the human hand. The union of these two parts, mechanics with the design of the prosthesis and electrical with the design of the device of acquisition and processing of EMG signals generated a nonconventional prosthesis directed to patients with transradial amputation type. Being the stage of acquisition and processing of signals, the most complex part of this experimentation, due to the low repeatability, as well as the external agents that are involved in the process. It is estimated that more complex control algorithm solved by genetic algorithms, can reduce the errors presented in the previous studies. ${ }^{21,22}$

In the aspect of the myoelectric signals, is convenient to design the intelligent control system that serves as a motion generator for the prosthesis. Since within this work only an electric pulse control system was proposed that feeds the servomotors. The main sources of noise that most interfere in the myoelectric signal measured in this research are related to the capacitive interference of the patient's body, to the measurement equipment produced by the power supply as well as the inductive interference caused mainly by the electric network, which produces magnetic fields that vary with the time, which in turn induced voltages in the network formed by the electrodes of the patient. According to Cifuentes, ${ }^{23}$ the filtering stage for myoelectric signals must consider analog filters in a frequency range of 10 to $500 \mathrm{~Hz}$, while $\mathrm{in}^{24}$ it is pointed out that a filter must be attached to reject notch bands of $50 \mathrm{0r} 60 \mathrm{~Hz}$ by means of Notch filter blocking the common signal from the mains. Another option according to ${ }^{25}$ for a later stage of this investigation is to use a fourth order active band pass filtering between 10 and $500 \mathrm{~Hz}$ for the purification of myoelectric signals.

\section{Acknowledgments}

The authors fully appreciate the support granted for this research work by Instituto Politécnico Nacional and all support given by CONACYT.

\section{Conflicts of interest}

The authors declare there is no conflict of interest.

\section{Funding details}

None.

\section{References}

1. Chávez Carmona MA, Rodríguez-Spitia Fy Baradica López A Exoskeletons to enhance human capabilities and support rehabilitation, Revista Ingeniería Biomédica. 2010;4(7):72-82.

2. Delgado-Saa JF, Vallejo E, Torres J. Design and construction of an acquisition and visualization system of electromyographic signals. Fifth LACCEI International Latin American and Caribbean Conference for Engineering and Technology. 2007. p. 1-6.

3. Borzelli D, Berger DJ, Pai DK, et al. Effort minimization and synergistic muscle recruitment for three-dimensional force generation. Front Comput Neurosci. 2013;7:186.

4. Criswell E. Cram's Introduction to Surface Electromyography, 2nd ed. Jones and Bartlett Publishers, 2011. P. 65-114.

5. Maier S, Van der Smagt P. Surface EMG suffices to classify the motion of each finger independently, 9th International Conference on Motion and Vibration Control. 2008.

6. De Luca C. Surface Electromyography: Detection and Recording. DelSys Incorporated. 2002. P. 1-10.

7. Moore KL, Dalley AF, Argur A. Anatomía con Orientación Clínica. 6th ed. Lippincott Williams \& Wilkins, 2007. P. 30-37.

8. Morillo DS, Ojeda JL, Foix LF, et al. Monitoring and analysis of cardio respiratory and snoring signals by using an accelerometer, 29th Conference Proceedings IEEE of Engineering in Medicine and Biology Society, 2007. P. 3942-3945.

9. Mohan M, Kumar RP, Agrawal R, et al. Finger vein recognition using Integrated Responses of Texture features. 4th International Work Conference on Bioinspired Intelligence. 2015. P. 209-214.

10. Ávila Morales CM, Gómez Cruz JM, Zapata Farfán J. Design and Construction of an Electromechanical Prosthesis of the Human Hand. Tesis de Licenciatura, Instituto Politécnico Nacional, Escuela Superior de Ingeniería Mecánica y Eléctrica unidad Zacatenco, 2013. P. 41-57.

11. Hargrove LJ, Englehart K, Hudgins B. A comparison of surface and intramuscular myoelectric signal classification. IEEE Emgineering in Medicine and Biology Society. 2007;54(5):847-853.

12. Romo HA, Realpe JC, Jojoa PE. Analysis of surface EMG signals and their application in hand prosthesis control. Revista Avances en Sistemas e Informática, 2007;4(1):127-136.

13. Zecca M, Micera S, Carroza MC, et al. Control of multifunctional prosthetic hands by processing the electromyographic signal, Critical Reviews in Biomedical Engineering. 2002;30(4-6):459-485.

14. Sarmiento Vela LC, Páez JJ. Sarmiento JF. Mechatronic prosthesis for people amputated between elbow and wrist. Tecné, Episteme y Didaxis. 2009;25:22-40.

15. Dorador González JM, Ríos Murillo P, Flores Luna I, et al. Robotics and smart prostheses. Revista Digital Universitaria UNAM. 2004;6(1):1-15.

16. Vélez CA. Design and construction of a prototype of biofeedback equipment for myography Miofeedback. Project-Control Miofeedback. 2009;2:15-21.

17. Seniam, The proyect Seniam. Recovered October 17, 2010.

18. Uribe-Londoño M, Saldarriaga-Fernández IC, Berna-Restrepo M, et al. Design and construction of an elbow joint controlled by myoelectric potentials. Revista CES Medicina, 2002;16(2):39-42.

19. Merletti R. Standards for reporting EMG data. Journal of Electromyography and Kinesiology. 1999;9(1):3-4.

20. Cifuentes González IA, Design and Construction of a System for the Detection of Electromyography Signals, Tesis Licenciatura, Universidad Autónoma de Yucatán, 2010. P. 1-110.

21. Khandpur RS. Biomedical Instrumentations: Technology and Applications, 1st ed. MacGraw-Hill, 2004. P. 22-96.

22. López N, Soria C, Orosco E, et al. Control mioeléctrico para movimiento en $2 \mathrm{D}$ de un manipulador robótico industrial. XVI Congreso Argentino de Bioingeniería, V Jornadas de Ingeniería Clínica. 2007. P. 595-598.

23. Vidal Silva C, Pavesi Farriol L. Desarrollo de un sistema de adquisición y tratamiento de señales electrocardiográficas. Revista de la Facultad de Ingeniería de la Universidad de Tarapacá. 2005;13(1):39-46. 
24. Khokhar Z, Xiao Z, Menon C. Surface EMG Pattern Recognition for Real-Time Control of a Wrist Exoskeleton. BioMedical Engineering Online. 2010;9:41-41.
25. López N, Soria C, Orosco E. Control mioeléctrico para movimiento en 2D de un manipulador robótico industrial. XVI Congreso Argentino de Bioingeniería, V Jornadas de Ingeniería Clínica. 2007. P. 595-598. 